\title{
Integrando Técnicas de Learning Analytics no processo de Gamificação em um Ambiente Virtual de Aprendizagem
}

\author{
Aline Nunes Ogawa ${ }^{1}$, Ana Carolina Tomé Klock ${ }^{2}$, Isabela Gasparini ${ }^{1,3,4}$ \\ ${ }^{1}$ Departamento de Ciência da Computação, ${ }^{3}$ PPGCA, ${ }^{4}$ PPGECMT, Universidade do \\ Estado de Santa Catarina (UDESC) - Joinville, SC - Brasil \\ ${ }^{2}$ Instituto de Informática, Universidade Federal do Rio Grande do Sul (UFRGS) - Porto \\ Alegre, RS - Brasil \\ alinenogawa@hotmail.com, actklock@inf.ufrgs.br, \\ isabela.gasparini@udesc.br
}

\begin{abstract}
Technologies as gamification and Learning Analytics (LA) have been applied to reduce the lack of interest and motivation problems in education. Gamification is the use of game elements in non-game context to motivate and engage students. LA seek to understand and improve the teaching-learning process to analyze the students and their contexts. The goal of work is to improve the gamification of a learning environment through comparative elements of gamification, seeking to increase the interaction and satisfaction of students. For this, a minicourse has been applied with two groups (control e experimental). The results show that there was not statistical difference between the groups in interaction, but the satisfaction was higher in experimental group.
\end{abstract}

Resumo. Tecnologias como a gamificação e as Learning Analytics (LA) têm sido utilizadas para reduzir os problemas de falta de interesse e motivação na educação. A gamificação é utilização de elementos de jogos em contexto não lúdicos para motivar e engajar os estudantes. As LA buscam entender $e$ otimizar o processo de ensino-aprendizagem para analisar os estudantes $e$ seus contextos. $O$ objetivo deste trabalho é melhorar a gamificação de um ambiente de aprendizagem por meio da adição de elementos comparativos da gamificação, buscando aumentar a interação e satisfação dos estudantes. Para isso, um minicurso foi aplicado com dois grupos (de controle e experimental). Os resultados indicam que não houve diferença estatística entre os grupos na interação, mas a satisfação foi maior no grupo experimental.

\section{Introdução}

Apesar da maioria dos estudantes crescer em meio às novas tecnologias e com métodos de ensino digitais (Prensky, 2001), muitos deles utilizam os Ambientes Virtuais de Aprendizagem (AVAs) apenas para acessar os conteúdos disponibilizados na disciplina e entregar atividades, havendo pouca ou nenhuma interação por parte do estudante (Falcão; Leite; Tenório, 2014). Esta falta de interação acaba os desmotivando e afetando diretamente o aprendizado e compreensão do conteúdo estudado. Com isso, a aplicação de novas estratégias para atrair o interesse dos estudantes e de tecnologias que possam 
VI Congresso Brasileiro de Informática na Educação (CBIE 2017)

Anais do XXVIII Simpósio Brasileiro de Informática na Educação (SBIE 2017)

ser aplicadas no processo de ensino-aprendizagem são necessárias. Entre estas abordagens e tecnologias, destacam-se a gamificação e as Learning Analytics (LA).

A gamificação é a utilização de características existentes em jogos com o intuito de motivar e engajar os estudantes (Kapp, 2012). Essa motivação pode ser definida como o estímulo que um indivíduo recebe para alcançar um objetivo ou meta específica, enquanto o engajamento é um comprometimento com uma causa maior (Ryan; Deci 2000). A gamificação pode influenciar três áreas (i.e., cognitiva, emocional e social) (Lee; Hammer, 2011) e, entre elas, destaca-se a área social, que se refere à comunicação, interação e comparação. Assim, a motivação dos estudantes pode aumentar se eles forem capazes de comparar o seu progresso individual com os seus colegas de turma (Vozniuk; Govaerts; Gillet, 2013).

Já as LA referem-se à interpretação de uma ampla gama de dados produzidos pelos estudantes a fim de avaliar o seu progresso acadêmico, prever o desempenho futuro e detectar possíveis problemas (Johnson et al., 2011). Elas permitem tanto ao professor quanto ao estudante visualizar o progresso e o desempenho individual e de toda a turma. Portanto, uma das formas de comparação é por meio da aplicação dos princípios de LA (i.e., a análise dos dados do estudante) aplicados à gamificação. Entretanto, essa integração ainda é pouco explorada pela literatura e a maioria dos ambientes se limita a apresentar a quantidade de pontos e as medalhas conquistadas em uma tabela de classificação.

A partir do exposto, o objetivo deste trabalho foi projetar, implementar e avaliar a integração de técnicas de LA com a gamificação em um AVA, buscando aumentar a interação e a satisfação do estudante durante um minicurso online de Algoritmos e Linguagem de Programação. Este trabalho está estruturado como segue: a Seção 2 descreve os conceitos fundamentais do trabalho. A Seção 3 apresenta a integração dos princípios de LA na gamificação, explicando os novos elementos de comparações individuais e entre os estudantes da turma. A Seção 4 explica o protocolo utilizado para realização de um experimento controlado, e a Seção 5 analisa e discute os resultados. Por fim, a Seção 6 apresenta as conclusões e trabalhos futuros.

\section{Fundamentos}

Gamificação é a utilização de elementos e design de jogos em contextos não lúdicos a fim de estimular e motivar seus usuários a alcançarem algum objetivo específico (Deterding et al., 2011; Werbach; Hunter, 2012). Os elementos de jogos mais comumente utilizados em ambientes educacionais são: pontos, emblemas (e.g., medalhas, troféus), missões, níveis, tabela de classificação, narrativa e avatar (Bissolotti; Nogueira; Pereira, 2014; Klock et al. 2014; Ogawa; Klock; Gasparini, 2016).

As LA são uma série de técnicas para medir, coletar, analisar e relatar os dados obtidos sobre os estudantes e seus contextos, com o intuito de entender e otimizar o processo de ensino e aprendizagem, bem como o ambiente em que este ocorre (Siemens, 2011). Um dos focos das LA é a análise de dados capturados automaticamente para estudar o comportamento dos estudantes (Agudo-Peregrina et al., 2014), buscando melhorar a aprendizagem por meio do acompanhamento de seu progresso e desempenho acadêmico. Entre as áreas das quais as LA mais emprestam suas técnicas, as mais 
aderentes a este trabalho foram: Estatística, e Visualização da Informação (InfoVis). Este trabalho utilizou técnicas de estatística e InfoVis para possibilitar a comparação dos elementos de jogos disponíveis na gamificação do AdaptWeb ${ }^{\circledR}$ (Ambiente de ensinoaprendizagem Adaptativo na $\mathrm{Web}$ ).

\section{Integração dos princípios de LA com a gamificação do AdaptWeb}

Este trabalho integrou princípios de LA com a gamificação existente no ambiente AdaptWeb $^{\circledR}$. Os princípios de LA (i.e., coleta e análise dos dados dos estudantes) utilizados são relativos às comparações que os estudantes podem fazer com sua turma, bem como visualizar seu andamento na disciplina (progresso e desempenho). A gamificação do AdaptWeb ${ }^{\circledR}$ é composta por: perfil do estudante, pontuação, desafios, tabela de classificação, transferência de moedas e loja. Cada um destes elementos é detalhado a seguir.

O Perfil do Estudante apresentava o total de pontos de experiência conquistado, o nível atual, o total de pontos necessários para subir para o próximo nível e uma barra de progresso que indica o percentual de conclusão do nível atual, conforme a Figura 1. Como elementos comparativos, foram incluídos o total de medalhas e de moedas conquistadas, bem como a posição do estudante na tabela de classificação. Assim, é possível acompanhar seu desempenho de forma objetiva.

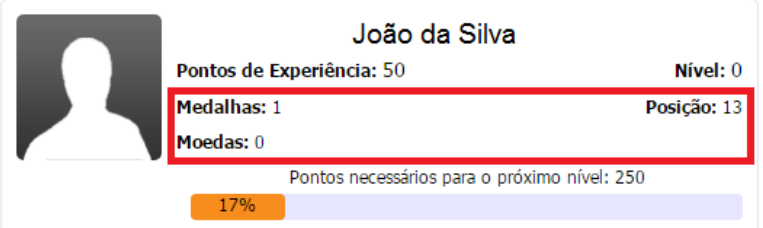

Figura 1. Perfil do estudante fictício

A Pontuação possui apenas elementos comparativos que possibilitam o estudante visualizar o seu progresso, comparando sua pontuação atual com a média de pontos da turma por meio de barras de progresso e apresentando a menor e a maior pontuação da turma nos seus extremos. Também é apresentado o histórico de pontos conquistados por atividade (e.g., acessar recursos, participar do fórum de discussão e enviar recados) e quantas vezes cada atividade foi realizada, conforme ilustra a Figura 2.

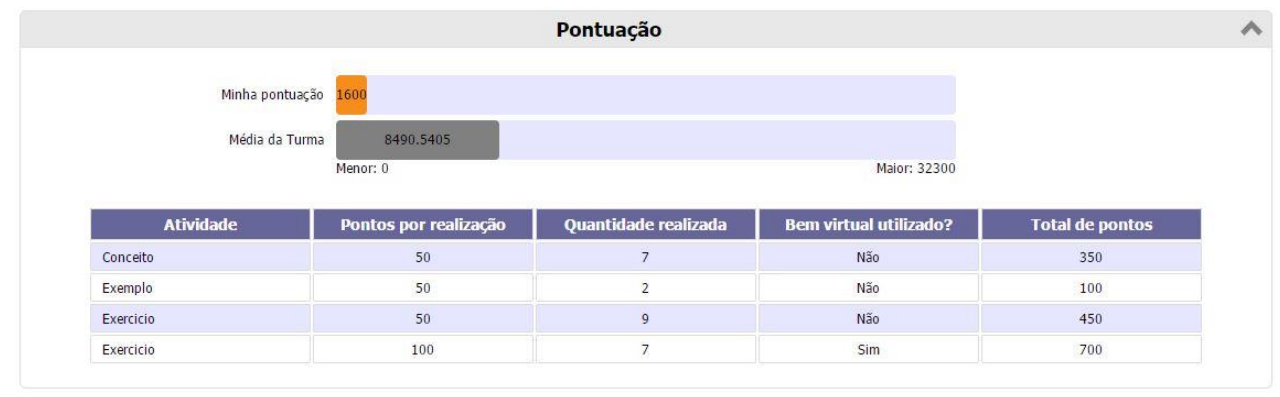

Figura 2. Pontuação do estudante

Os Desafios descreviam as missões que deveriam ser completadas para obter as vinte medalhas disponíveis. Foram incluídas informações relacionadas ao total de medalhas que o estudante já conquistou (por meio de uma barra de progresso) e quantos 
VI Congresso Brasileiro de Informática na Educação (CBIE 2017)

Anais do XXVIII Simpósio Brasileiro de Informática na Educação (SBIE 2017)

estudantes já tinham conquistaram cada medalha. A Figura 3 exemplifica os desafios, onde as medalhas conquistadas eram apresentadas coloridas e as disponíveis (i.e., não conquistadas) em tons de cinza.

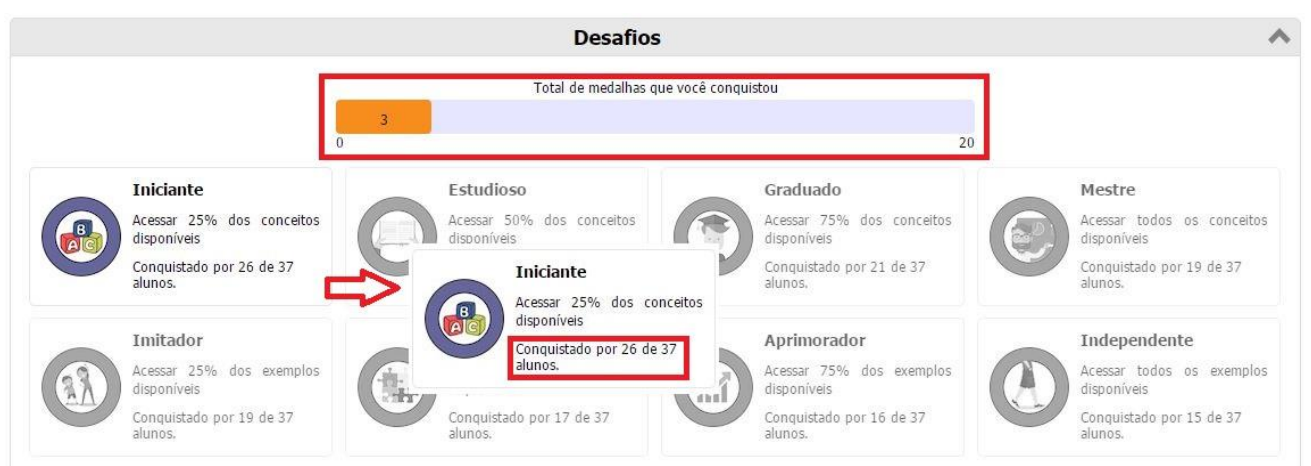

Figura 3. Desafios disponíveis no AdaptWeb ${ }^{\circledR}$

A Tabela de Classificação apresentava a posição de cada estudante, seu avatar, seu nome, o total de pontos conquistados, seu nível e os desafios completados. A posição do estudante na tabela de classificação fica destacada, conforme Figura 4. Não foram adicionados elementos comparativos para este caso.

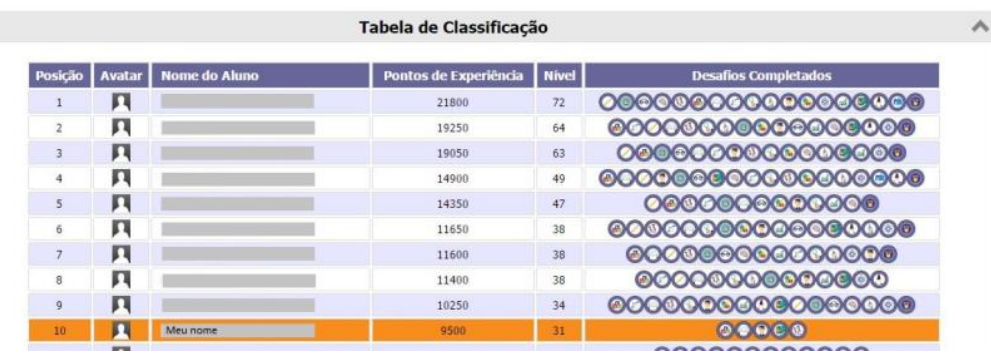

Figura 4. Tabela de classificação

A Transferência de Moedas: possibilitava a doação de moedas entre os estudantes e a visualização do histórico de doações. Foram acrescentados os totais de moedas doadas e recebidas de modo a facilitar a sua visualização, conforme Figura 5.

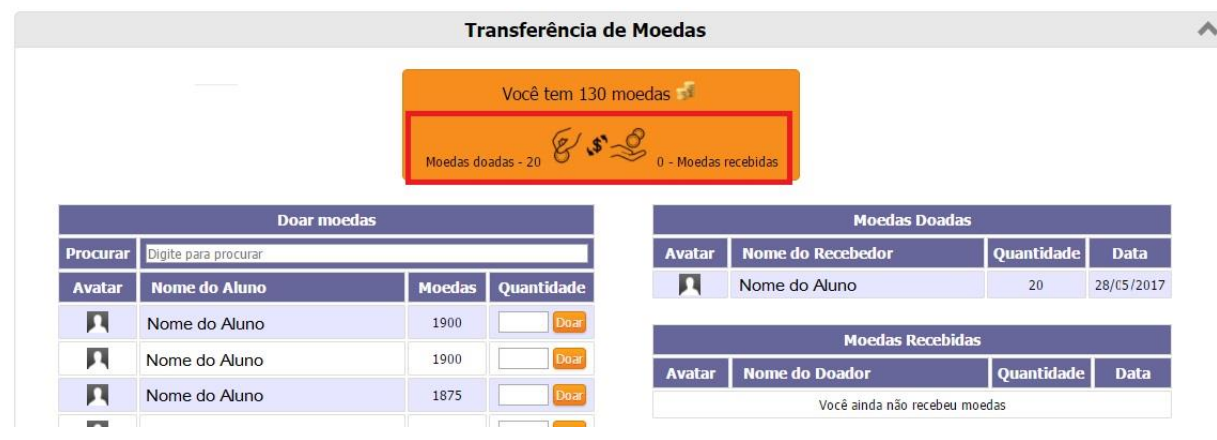

Figura 5. Transferência de moedas

A Loja apresentava quatro bens virtuais e o histórico de compras. Foi incluído o total de vezes que o estudante comprou cada bem, conforme a Figura 6. 
VI Congresso Brasileiro de Informática na Educação (CBIE 2017)

Anais do XXVIII Simpósio Brasileiro de Informática na Educação (SBIE 2017)

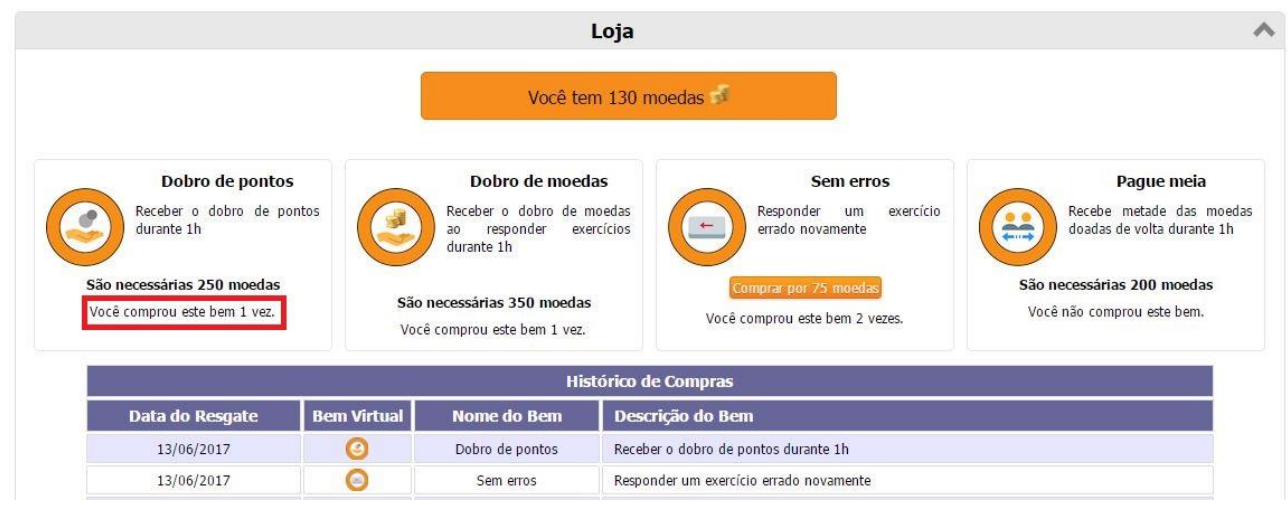

Figura 6. Loja

A Tabela 1 apresenta as formas de comparação sem e com a implementação deste trabalho. Anteriormente, o estudante podia comparar seu progresso e desempenho dos pontos e medalhas em relação à turma somente por meio da tabela de classificação, não havendo uma análise detalhada. Com este trabalho, tornou-se possível para que estudantes tenham conhecimento do seu progresso e seu desempenho por meio da comparação dos pontos com a turma, do detalhamento dos pontos ganhos, das análises dos desafios (i.e., total de medalhas conquistadas e quantidade de alunos que conquistaram cada medalha), das moedas doadas e recebidas e do total de compras de cada bem virtual. A análise dos bens virtuais e das moedas existia por meio do histórico destas, mas, segundo avaliações anteriores, necessitava de mais clareza e detalhamento. Desta forma, a inclusão acrescentou-se de forma clara tais informações.

Tabela 1: Formas de comparação dos elementos de jogos

\begin{tabular}{|c|c|c|}
\hline Elementos de jogos & $\begin{array}{l}\text { AdaptWeb }{ }^{\circledR} \\
\text { Sem este trabalho }\end{array}$ & $\begin{array}{l}\text { AdaptWeb }{ }^{\circledR} \\
\text { Com este trabalho }\end{array}$ \\
\hline Pontos & Tabela de classificação & $\begin{array}{l}\text { Tabela de classificação; } \\
\text { Comparação dos pontos; } \\
\text { Detalhamento de pontos ganhos } \\
\text { conforme o tipo de atividade }\end{array}$ \\
\hline Emblemas & Tabela de classificação & $\begin{array}{l}\text { Tabela de classificação; } \\
\text { Total de medalhas conquistadas; } \\
\text { Total de alunos que conquistaram } \\
\text { cada medalha }\end{array}$ \\
\hline Moedas & Histórico & $\begin{array}{l}\text { Histórico; } \\
\text { Total de moedas doadas e recebidas }\end{array}$ \\
\hline Bens Virtuais & Histórico & $\begin{array}{l}\text { Histórico; } \\
\text { Total de compra do bem virtual }\end{array}$ \\
\hline
\end{tabular}

Para testar a inclusão destes novos elementos foi realizado um experimento controlado, onde os estudantes foram divididos em dois grupos: experimental e de controle. $\mathrm{O}$ grupo de controle utilizou a gamificação já existente, enquanto o grupo experimental utilizou o ambiente com os princípios de LA incorporados. 


\section{Experimento Controlado}

Um minicurso online de Algoritmos e Linguagem de Programação foi disponibilizado para os estudantes de graduação da Universidade do Estado de Santa Catarina (UDESC). Este tema foi escolhido devido ao alto índice de reprovação e por ser uma disciplina obrigatória da grade curricular de 8 dos 9 cursos ofertados na área de ciências, engenharias e tecnologias.

O período de matrícula no minicurso foi entre os dias 23 e 27/05/2017. A matrícula era efetivada após o estudante: 1) se cadastrar no ambiente; 2) responder o Levantamento de Perfil; 3) aceitar o Termo de Consentimento Livre e Esclarecido (TCLE), que permitia o uso dos dados capturados e informava sobre os procedimentos do minicurso; e 4) o Questionário de Tipo de Jogador. Em seguida, os estudantes foram divididos em dois grupos: de controle e experimental. A divisão dos grupos possibilitou a distribuição dos alunos da forma mais igualitária possível, com base nos seguintes dados: professor, curso, sexo e idade.

O minicurso ficou disponível de 29/05 até 04/06, permitindo que os estudantes acessassem os mais de 200 objetos de aprendizagem disponíveis (entre conceitos, exemplos, materiais complementares, links de apoio, exercícios, além do fórum de discussão e do mural de recados). Durante esse período, foram realizadas intervenções diárias por e-mail e via mural de recados, e lançados desafios no fórum de discussão para ambos os grupos. Entre dias 05 e 06/06, o sistema disponibilizou a avaliação final e o questionário de satisfação.

\section{Análise e Discussão dos Resultados}

Dos 77 estudantes inscritos no minicurso, 45 (58\%) eram do sexo masculino e 32 (32\%) do sexo feminino. Em relação à idade, a Figura 7 apresenta a distribuição de idades dos estudantes, no qual é possível observar que 49\% dos estudantes têm entre 18 e 19 anos. Já o gráfico de distribuição dos cursos é apresentado na Figura 8. Nele é possível observar que metade dos estudantes é dos cursos de Ciência da Computação e Engenharia Mecânica.

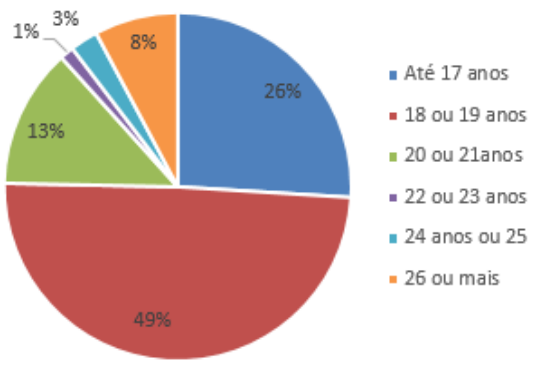

Figura 7. Distribuição de idade

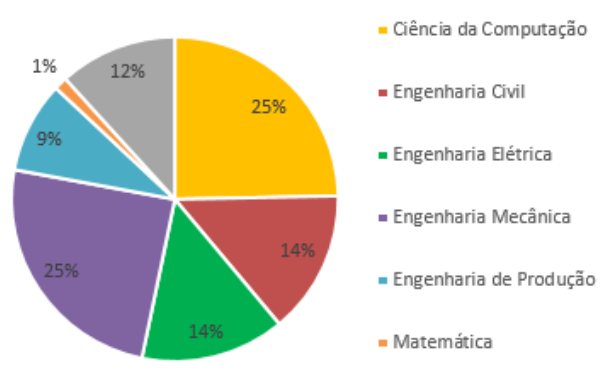

Figura 8. Distribuição de curso

Para a análise de interação, foram verificados os totais de acessos aos conceitos e exemplos, de exercícios resolvidos, de recados enviados, de participação no fórum de discussão, o desempenho nos exercícios e na avaliação. A Tabela 2 apresenta o total de estudantes que acessaram os recursos pelo menos uma vez, a soma de suas interações e a média, independente de terem acessado ou não a gamificação. 
VI Congresso Brasileiro de Informática na Educação (CBIE 2017)

Anais do XXVIII Simpósio Brasileiro de Informática na Educação (SBIE 2017)

Tabela 2: Interação dos Estudantes

\begin{tabular}{|l|c|c|c|c|c|c|}
\cline { 2 - 8 } \multicolumn{1}{c|}{} & \multicolumn{3}{c|}{ Grupo de Controle } & \multicolumn{3}{c|}{ Grupo Experimental } \\
\hline Dado analisado & Amostra & Interação & Média & Amostra & Interação & Média \\
\hline Acesso aos conceitos & 30 & 1186 & 39,55 & 34 & 1022 & 30,06 \\
\hline Acesso aos exemplos & 23 & 644 & 28,00 & 21 & 527 & 25,09 \\
\hline Exercícios resolvidos & 26 & 637 & 24,50 & 16 & 461 & 28,81 \\
\hline Mensagens no Mural & 3 & 4 & - & 1 & 1 & - \\
\hline $\begin{array}{l}\text { Tópicos criados ou } \\
\text { respondidos no } \\
\text { fórum de discussão }\end{array}$ & 7 & 12 & 1,71 & 7 & 8 & 1,14 \\
\hline Exercícios corretos & 25 & 477 & 19,08 & 15 & 343 & 22,86 \\
\hline Nota na avaliação & 27 & 219 & 8,11 & 26 & 193 & 7,42 \\
\hline
\end{tabular}

Com a Tabela 2 é possível observar que, apesar de ter mais estudantes do grupo de controle terem acessado o ambiente, a média de interações na resolução e acerto dos exercícios foi maior no grupo experimental.

A Tabela 3 apresenta as interações considerando somentes os estudantes que utilizaram a gamificação. Por meio dela, é possível observar que havia mais estudantes do grupo de controle. No entanto, a média de resolução e acertos nos exercícios, e acessos aos exemplos foi maior no grupo experimental.

Tabela 3: Interação dos Estudantes que acessaram a gamificação

\begin{tabular}{|l|c|c|c|c|c|c|}
\cline { 2 - 8 } \multicolumn{1}{c|}{} & \multicolumn{3}{c|}{ Grupo de Controle } & \multicolumn{3}{c|}{ Grupo Experimental } \\
\hline Dado analisado & Amostra & Interação & Média & Amostra & Interação & Média \\
\hline Acesso aos conceitos & 16 & 773 & 48,31 & 10 & 381 & 38,1 \\
\hline Acesso aos exemplos & 14 & 494 & 35,29 & 7 & 341 & 48,71 \\
\hline Exercícios resolvidos & 17 & 464 & 27,29 & 9 & 284 & 31,56 \\
\hline Mensagens no Mural & 3 & 4 & - & 1 & 1 & - \\
\hline $\begin{array}{l}\text { Tópicos criados ou } \\
\text { respondidos no } \\
\text { fórum de discussão }\end{array}$ & 6 & 11 & 1,83 & 5 & 6 & 1,20 \\
\hline Exercícios corretos & 17 & 254 & 14,94 & 9 & 215 & 23,89 \\
\hline Nota na avaliação & 18 & 147 & 8,17 & 12 & 95 & 7,92 \\
\hline
\end{tabular}

Para verificar se havia diferença estatística entre os grupos, foram aplicados os testes de Shapiro-Wilk (para verificar a normalidade dos dados) e de Mann-Whitney U (para o teste de significância, uma vez que a amostra não possuía distribuição normal). Não foram constatadas diferenças significativas para nenhum caso: considerando todos os estudantes (Tabela 1) e somente os que acessaram a gamificação (Tabela 2).

Em seguida, a satisfação foi analisada por meio da aplicação de um questionário de satisfação. Dos 26 estudantes do grupo de controle, 12 responderam ter utilizado a gamificação, e dos 25 do grupo de controle, apenas 8. No entanto, 5 do grupo de controle e 2 do grupo experimental utilizaram, apesar de responderem que não e um do grupo de controle que afirmou ter usado, mas não utilizou. As principais justificativas dos estudantes por não terem acessado a gamificação foram a falta de tempo e de duração do minicurso (que foi de apenas 7 dias), enquanto o principal motivo para a 
VI Congresso Brasileiro de Informática na Educação (CBIE 2017)

Anais do XXVIII Simpósio Brasileiro de Informática na Educação (SBIE 2017)

acessarem foram o acompanhamento do progresso, do desempenho e dos elementos de jogos.

Tabela 4: Influência da gamificação

\begin{tabular}{|l|c|c|c|c|c|c|c|c|c|}
\hline \multirow{2}{*}{ Influência em: } & \multicolumn{2}{|c|}{ Interação } & \multicolumn{2}{c|}{ Comunicação } & \multicolumn{2}{c|}{ Desempenho } & \multicolumn{2}{c|}{ Satisfação } \\
\cline { 2 - 11 } & $\mathrm{C}$ & $\mathrm{E}$ & $\mathrm{C}$ & $\mathrm{E}$ & $\mathrm{C}$ & $\mathrm{E}$ & $\mathrm{C}$ & $\mathrm{E}$ \\
\hline Acesso aos recursos & $=$ & + & $=$ & $=+$ & + & + & + & + \\
\hline $\begin{array}{l}\text { Acompanhamento do } \\
\text { progresso }\end{array}$ & $=$ & + & $=$ & + & + & + & + & + \\
\hline Personalização da foto & $=$ & $=$ & $=$ & $=$ & $=$ & $=$ & $=$ & $=$ \\
\hline Tarefas & + & $=$ & + & $=$ & + & + & + & + \\
\hline $\begin{array}{l}\text { Comparação com } \\
\text { outros alunos }\end{array}$ & + & $=+$ & + & $=$ & + & + & + & + \\
\hline $\begin{array}{l}\text { Responder os } \\
\text { exercícios }\end{array}$ & + & $=$ & + & $=$ & + & + & + & + \\
\hline Doação de moedas & $=$ & $=$ & $=$ & $=$ & $=$ & + & $=$ & + \\
\hline Compra de itens & $=$ & $=$ & $=$ & $=$ & $=+$ & + & $=+$ & + \\
\hline
\end{tabular}

Os estudantes que utilizaram a gamificação também responderam perguntas referentes a influência dos elementos de jogos na interação, comunicação, desempenho e satisfação, sendo desconsiderado o estudante que não utilizou. A Tabela 3 apresenta se a influência foi negativa (representada por “-"), neutra (representada por "=”) ou positiva (representada por “+”), para os grupos de controle (representado por "C") e experimental (representado por "E"). O valor corresponde a média de todas as respostas dos estudantes.

Como é possível observar, a interação e a comunicação tiveram mais respostas neutras, mas o grupo de controle teve mais respostas de influências positivas se comparado ao grupo experimental. Já o desempenho e a satisfação tiveram influências positivas para a maioria dos elementos de jogos, tendo mais respostas positivas no grupo experimental.

Além disso, os itens que mais agradaram os estudantes foram o conteúdo e o auxílio na disciplina (presencial) do seu curso e os que menos agradaram foi o conteúdo (especificamente o último tópico, que poderia ser mais detalhado) e o tempo, visto que a duração do minicurso foi de uma semana e nem todos tiveram tempo disponível para utilizar o ambiente.

Por fim, foram analisadas as doações de moedas, as medalhas conquistadas e as compras de bens virtuais. Apenas um estudante fez doação de moedas, pertencente grupo de controle. As medalhas foram conquistadas por 26 estudantes, 15 do grupo de controle e 11 do grupo experimental, que completaram 186 e 114 desafios respectivamente. Quanto aos bens virtuais, 7 estudantes compraram bens virtuais, 5 do grupo de controle (8 compras) e 2 do grupo experimental (27 compras). Destaca-se que, apesar de o grupo experimental ter menos estudantes, estes compraram mais vezes.

\section{Conclusão}

A gamificação e as LA vem sendo utilizadas no contexto educacional para tentar reduzir os problemas de falta de motivação e interesse dos alunos. Quando os princípios de LA 
são integrados à gamificação, a visualização do progresso e desempenho de cada estudante é facilitada, bem como a comparação com os demais estudantes da turma.

O objetivo deste trabalho foi projetar, implementar e avaliar a integração de técnicas de LA com a gamificação em um AVA, buscando aumentar a interação e a satisfação dos estudantes. Para avaliar este objetivo foi realizado um minicurso online de Algoritmos e Linguagem de Programação, que contou com 77 estudantes inscritos. Os estudantes eram predominantemente do sexo masculino $(58 \%)$ com idade entre 18 e 19 anos (49\%) e dos cursos de Ciência da Computação (25\%) e Engenharia Mecânica (25\%). Para a análise de interação, apenas aqueles que acessaram pelo menos uma vez os recursos foram considerados na análise. Apesar dos estudantes do grupo de controle acessarem mais recursos, a média de resolução e de acertos nos exercícios foi maior para o grupo experimental. Para verificar a normalidade e a significância, foram aplicados os testes estatísticos, mas não foi possível constatar nenhuma diferença significativa considerando o ambiente e a utilização dos elementos de jogos comparativos.

Por meio de um questionário de satisfação foi identificado que a gamificação teve influência neutra na interação e na comunicação e positiva no desempenho e na satisfação dos estudantes. A satisfação foi maior no grupo experimental, que não considerou a influência positiva apenas para um elemento de jogos. Quanto à utilização dos elementos de jogos, o grupo de controle fez doação de moedas e conquistou mais medalhas, enquanto o grupo experimental comprou mais bens virtuais.

Portanto, constatou-se que houve aumento de interação apenas na resolução e acerto dos exercícios com os elementos comparativos adicionados, porém a satisfação foi maior para os estudantes do grupo de controle. Ambos resultados não foram validados de forma estatisticamente significativa. Como trabalhos futuros outros experimentos devem ser realizados, com maior duração, para verificar se o tempo foi um fator importante para a análise. Além disso, uma investigação maior junto aos estudantes, de forma qualitativa, deve ser explorada. Outras técnicas de LA também podem trazer benefícios para o ambiente de gamificação e serão exploradas em outros trabalhos.

\section{Referências}

Agudo-Peregrina, Á. F.; Iglesias-Pradas, S.; Conde-González, M. Á.; Hernández-García, Á. (2014). Can we predict success from log data in VLEs? Classification of interactions for learning analytics and their relation with performance in VLEsupported F2F and online learning. Computers in human behavior, v. 31, p. 542-550.

Bissolotti, K.; Nogueira, H. G.; Pereira, A. T. C. (2014). Potencialidades das mídias sociais e da gamificação na educação a distância. In: RENOTE - Revista de Novas Tecnologias na Educação, v. 12, n. 2.

Deterding, S.; Dixon, D.; Khaled, R.; Nacke, L. (2011). From game design elements to gamefulness: defining gamification. In: Proceedings of the 15th international academic MindTrek conference: Envisioning future media environments. p. 9-15. 
VI Congresso Brasileiro de Informática na Educação (CBIE 2017)

Anais do XXVIII Simpósio Brasileiro de Informática na Educação (SBIE 2017)

Falcão, A. P.; Leite, M. D.; Tenório, M. M. (2014) Ferramenta de apoio ao ensino presencial utilizando gamificação e design de jogos. In: Simpósio Brasileiro de Informática na Educação (SBIE), p. 526-533.

Johnson, L.; Smith, R.; Willis, H.; Levine, A.; Haywood, K. (2011). The NMC Horizon Report: 2011 Higher Education Edition. New Media Consortium.

Kapp, K. M. (2012). The Gamification of Learning and Instruction: Gamebased methods and strategies for training and education. San Francisco: John Wiley \& Sons.

Klock, A. C. T.; Carvalho, M. F. D.; Rosa, B. E.; Gasparini, I. (2014). Análise das técnicas de Gamificação em Ambientes Virtuais de Aprendizagem. RENOTE Revista Novas Tecnologias na Educação, v. 12, n. 2, p. 1-10.

Lee, J. J.; Hammer, J. (2011). Gamification in education: What, how, why bother? Academic exchange quarterly, v. 15, n. 2, p. 146.

Ogawa, A. N.; Klock, A. C. T.; Gasparini, I. (2016). Avaliação da gamificação na área educacional: um mapeamento sistemático. In: Simpósio Brasileiro de Informática na Educação (SBIE), p. 440-449.

Prensky, M. (2001). Digital natives, digital immigrants part 1. On the horizon, v. 9, n. 5, p. 1-6.

Ryan, R. M.; Deci, E. L. (2000). Intrinsic and extrinsic motivations: Classic definitions and new directions. Contemporary Educational Psychology, v. 25, n. 1, p. 54-67.

Siemens, G. Learning Analytics \& Knowledge: February 27-March 1, 2011 in Banff, Alberta. 2010. Disponível em: <https://tekri.athabascau.ca/analytics/>. Acesso em: 10 agosto 2016.

Vozniuk, A.; Govaerts, S.; Gillet, D. (2013). Towards portable learning analytics dashboards. In: 2013 IEEE 13th International Conference on Advanced Learning Technologies. p. 412-416.

Werbach, K.; Hunter, D. (2012). For the win: How game thinking can revolutionize your business. Wharton Digital Press. 